\title{
Band gap structure of elliptic rods in water for a 2D phononic crystal
}

\author{
Oral Oltulu ${ }^{1} \cdot$ Amirullah M. Mamedov ${ }^{2,3} \cdot$ Ekmel Ozbay $^{2}$
}

Received: 1 August 2016/ Accepted: 27 December 2016 / Published online: 3 March 2017

(C) Springer-Verlag Berlin Heidelberg 2017

\begin{abstract}
The propagation of acoustic waves in twodimensional sonic crystals (SC) is studied theoretically. Effects of elliptical rod orientations on the acoustic band gaps in periodic arrays of rigid solid rods embedded in a polar liquid are investigated. We have found that the pass bands and forbidden bands of the sonic crystals can be changed by utilizing the rotational anisotropy of the structure factor at different rotation angles of the scatterers. The plane wave expansion (PWE) method is used to calculate the band structure. The variation of the absolute band gap was also investigated as a function of any filling fraction at a fixed orientation of the elliptical columns. The gap-tuning effect can be controlled by the rotational asymmetry and eccentricity of the scatterers.
\end{abstract}

\section{Introduction}

The propagation of acoustic waves in a composite medium has been an interesting topic for the last decade mainly due to its technological applications in condensed matter physics, especially in phononics [1, 2]. Since their invention, phononic crystals have triggered considerable interest because of their unusual acoustical properties. The unique properties of phononic crystals open up new and

Amirullah M. Mamedov

mamedov46@gmail.com

1 Department of Physics, Faculty of Science and Letters, Harran University, Sanliurfa, Turkey

2 Nanotechnology Research Center (NANOTAM), Bilkent University, Ankara, Turkey

3 International Scientific Center, Baku State University, Baku, Azerbaijan major fields in acoustics. Sonic crystals (SCs) are defined as structured materials formed by a periodic distributions of acoustic scatterers embedded in a host material, with strong periodic modulations in their density and elastic coefficients between the constituent materials. The periodicity of the scatterers in a surrounding material gives rise to the appearance of sonic band gaps, a range of frequencies for which sound propagation is forbidden inside the crystal. These stop bands were observed at frequencies depending on the lattice constant of the array in the band structures of crystals with various lattice geometries [3-5]. The SCs have induced several application proposals and enabled development of many new technologies. Recent studies in this field have attracted a great interest due to the splendid application prospects, such as acoustic filters [6, 7], shield devices [8-10], defect states for acoustic filters and wave guides [11], and so on. Recently, an increased attenuation at low frequencies has been achieved by the locally resonant sonic materials formed by soft and rigid elements [12]. Several theoretical methods have been used to study the elastic/acoustic band structures, such as, the plane-wave expansion (PWE) method [13, 14], the finite difference time domain (FDTD) method [15], the extended plane wave expansion (EPWE) method $[16,17]$, the multiple scattering theory (MST) [18], perturbative approach [19], and variational method [20]. Among them, the PWE is the most widely used method for calculating the band structures. In the literature, acoustic band gaps of various two-dimensional sonic crystal structures were investigated and these structures mostly consist of circular cross-section scatterers in square, triangular, rectangular lattices, the constituent being either both solids or fluids, or mixed solid-fluid [21]. Practically, band gaps properties of sonic crystal structures or the acoustic dispersion of the branches can be controlled by the lattice filling factor, the constituent 
material contrast between the rods and the surrounding, and the lattice symmetry, and as well as the scatter shape [22]. However, there are fewer studies that consider the shape of the inclusions despite the fact that shape factor is an important quantity for the propagation of acoustic waves in $2 \mathrm{D}$ phononic crystals [23-25]. Moreover, most of the work in this field focuses on the calculations of the band structure of 2D phononic crystals of circular rods.

In the present paper, the propagation of audible acoustic waves in two-dimensional periodic arrays of elliptic rigid solid rods embedded in polar liquids is considered. We have investigated the effects of orientations of elliptic rods on the acoustic band gaps. As a choice of material, we have considered polar liquids and perovskite-type materials. The speed of sound and the conductivity in most polar liquids increases with temperature [26]. On the other hand, the perovskite-type materials have been the subject of ongoing research for over 50 years. $\mathrm{ABO}_{3}$-type perovskite crystals are used extensively for technological applications. In this study, we have chosen water as a polar liquid and a well-known classic ferroelectric $\mathrm{BaTiO}_{3} \cdot \mathrm{BaTiO}_{3}$ is a promising material that has a wide range of applications in dielectric capacitors, pyroelectric detectors, nonlinear optical devices, phononic crystals, and surface-acoustic wave devices [27-31]. There are several reasons why $\mathrm{BaTiO}_{3}$ has been chosen as a material of interest. $\mathrm{BaTiO}_{3}$ is insoluble in alkalis and water, and durable material. It also shows captivating features with temperature. The material exhibits interesting self-assembly properties by changing its structure at different temperatures from below about -70 to above $120^{\circ} \mathrm{C}$. For example, while $\mathrm{BaTiO}_{3}$ is paraelectric with a cubic structure at temperatures higher than $120^{\circ} \mathrm{C}$, It forms a stable tetragonal structure at room temperature. Below $0^{\circ} \mathrm{C}$ it has a stable orthorhombic structure. Therefore, it may be interesting to see how the band structure is affected in the transition from one stable structure to another. However, although $\mathrm{BaTiO}_{3}$ is probably the most extensively studied material, surprisingly, there are not much data available on elastic properties of $\mathrm{BaTiO}_{3}$. Hence, the growing interest in phononic crystals requires new materials and material properties.

\section{Theory}

In this study, we consider a two-dimensional periodic composite that composed of solid elliptic rods immersed in polar liquids. Since mixed fluid/solid model can be simplified by imposing elastic rigidity to the solid media, the solid can be reasonably approximated as fluid inclusions. Then, the model allows only the longitudinal wave to propagate through the fluid media. Therefore, the acoustic wave equation in a sonic crystal can be written as a second order inhomogeneous differential equation in the form of pressure waves:

$\left(\rho c^{2}\right)^{-1} \frac{\partial^{2} p}{\partial t^{2}}=\vec{\nabla} \cdot\left(\rho^{-1} \nabla p\right)$,

where $p$ is the pressure, $\left(\rho c_{l}^{2}\right)^{-1}$ is the longitudinal elastic constant $C_{11}$, and $\rho$ is the mass density, and $\nabla(x, y)$ is the two-dimensional Nabla. Solution of this equation gives the band-gap structure of sonic crystals by the plane-wave expansion (PWE) method. According to the PWE method, $\rho^{-1}$ and $\left(\rho c^{2}\right)^{-1}$ can be expanded as material parameters in two-dimensional Fourier series [32]. Hence, the Fourier series expansion for $\mathrm{C}_{11}$ and $\rho$ can be expressed as

$\left(\rho c_{l}^{2}\right)^{-1}(\vec{r})=\sum_{\vec{G}} \eta^{-1}(\vec{G}) e^{i \vec{G} \cdot \vec{r}},(\rho)^{-1}(\vec{r})=\sum_{\vec{G}} \sigma^{-1}(\vec{G}) e^{i \vec{G} \cdot \vec{r}}$

The Fourier expansion coefficients for the inverse of mass density

$\sigma^{-1}(\vec{G})=\frac{1}{A_{\mathrm{c}}} \int \rho^{-1}(\vec{r}) d^{2} r \begin{cases}\rho_{a} f+\rho_{b}(1-f) & \text { for } \vec{G}=0 \\ \left(\rho_{a}-\rho_{b}\right) S(\vec{G}) & \text { for } \vec{G} \neq 0,\end{cases}$

where $A_{c}$ is the area of the unit cell, $f$ is the filling fraction, describing the ratio of the surface area of the column to the area of the total unit cell, the subindex " $a$ " and " $b$ " stands for the scatters and background material, respectively. $S(G)$ term in the equation is the structure factor defined for the system of elliptic rods with rotation angle $\psi$ in two-dimensional system as [33]

$S_{\mathrm{RC}}(\vec{G})=2 f \frac{J_{1}\left(\sqrt{r_{x}^{2} G_{x}^{\prime 2}+r_{x}^{2} G_{x}^{\prime 2}}\right)}{\sqrt{r_{x}^{2} G_{x}^{\prime 2}+r_{x}^{2} G_{x}^{\prime 2}},}$

where $G_{x}{ }^{\prime}$ and $G_{y}{ }^{\prime}$ are the vectors in the primed system while $\Gamma_{x}$ and $G_{y}$ are the $\mathrm{G}$ vector components in the original coordinate system, $J_{1}(x)$ is the Bessel function of the first kind of order one, $r_{y}$ and $r_{x}$ are the half length of the long and the short axis, respectively. The new $\mathrm{G}$ vector in primed coordinates can be expressed more compactly, in matrix form, as

$\left(\begin{array}{c}G_{x}^{\prime} \\ G_{y}^{\prime}\end{array}\right)=\left(\begin{array}{cc}\cos \theta & \sin \theta \\ -\sin \theta & \cos \theta\end{array}\right)\left(\begin{array}{c}G_{x} \\ G_{y}\end{array}\right)$

where the angle $\theta$ is defined as the angle between the long axis and $k x$ direction. 


\section{Results and discussion}

The model discussed here is shown in Fig. 1. Elliptical columns in water were arranged in a square lattice. In general, the band gap properties of phononic or sonic crystals are controlled by the lattice type, geometry, material density, speed of sound, and the filling ratio. In this study, we fix the material parameters for the elliptic rods in the sonic crystal and rotate the rods in the polar liquid media to extract the dependence of the sonic properties. $\mathrm{BaTiO}_{3}$ is chosen as the material for the elliptical inclusions. The mass density and sound speeds of $\mathrm{BaTiO}_{3}$ and polar liquid (water) are as follows: $\rho=5550 \mathrm{~kg} / \mathrm{m}^{3}, c_{1}=5640 \mathrm{~m} / \mathrm{s}$, for $\mathrm{BaTiO}_{3}$; $\rho=1000 \mathrm{~kg} / \mathrm{m}^{3}, c_{1}=1500 \mathrm{~m} / \mathrm{s}$ for water, respectively. The lattice constant of the sonic crystal is $a_{\mathrm{o}}=13 \mathrm{~mm}$, and the long axis of elliptic rods $r_{x}=0.3 a_{\mathrm{o}} \mathrm{mm}$ and the short axis is $r_{y}=0.5 a_{\mathrm{o}} \mathrm{mm}$. Numerical calculations based on these equations were performed by the code written by Elford [34] after some modifications.

Figure 2 shows the band structure for $\mathrm{BatiO}_{3}$ columns in water for the constant filling fraction angles.

For a square lattice with a lattice constant of $a_{o}$, the filling fraction $f$ is given by $f=r_{x} r_{y} \pi / A_{c}$, where $A_{\mathrm{c}}$ is the area of the unit cell. The results shown in Fig. 2 are for constant filling fraction of $\mathrm{BaTiO}_{3}=0.32$ for two different rotation angles of (a) $\theta=-45^{\circ}$, and (b) $\theta=45^{\circ}$. From Fig. 2a, it is seen that the absolute sonic band gap does not exist at $\theta=-45^{\circ}$. The non-existent bands at $\theta=-45^{\circ}$ start to open up at a positive angle of $45^{\circ}$. Therefore, it can be concluded that the gaps only appear at certain angles. This is simply due to rotational anisotropy of the structure factor at these angles.

When a band-gap has been found for a sonic crystal, the acoustic sound waves can be confined in a waveguide. In such systems, it is convenient and usually customary to express the absolute band gap widths in dimensionless units to make the gap scale independent of crystal size. The size of the photonic band gap can be characterized by expressing the quantity in terms of the mid-gap to band gap ratio. This ratio is defined as $\Delta \omega / \omega_{\mathrm{g}}$ [35], where $\Delta \omega$ is the frequency width of the gap and $\omega_{\mathrm{g}}$ is the

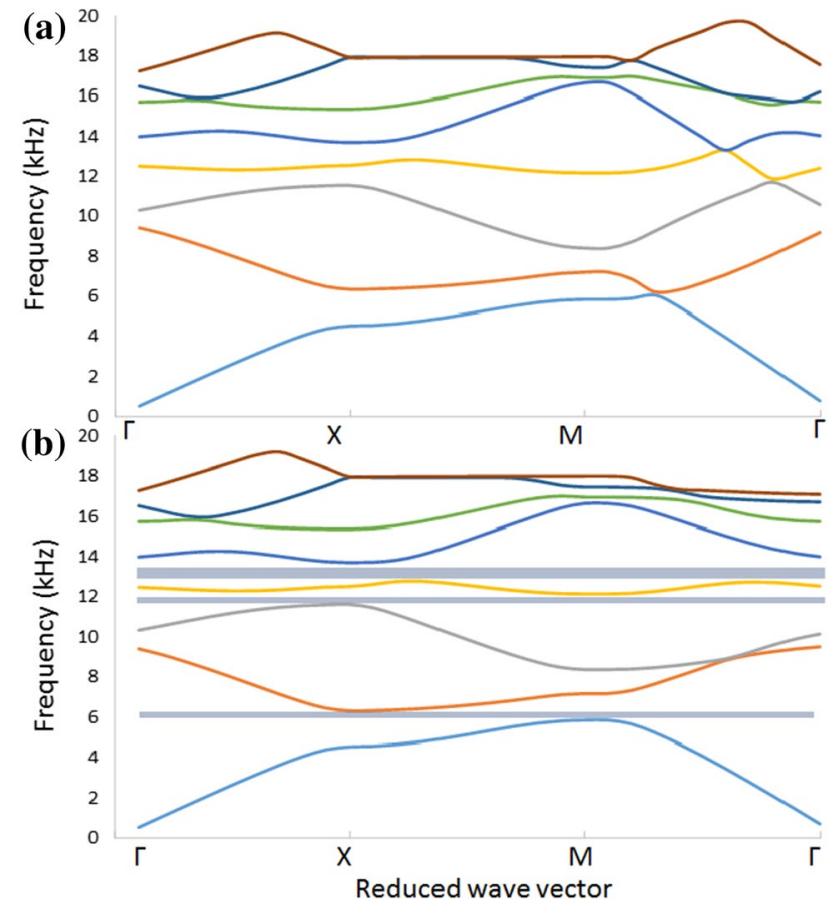

Fig. 2 Band structure for elliptical rods in polar liquid water at filling fraction $f=0.32$ at $\mathbf{a} \theta=-45^{\circ}, \mathbf{b} \theta=45^{\circ}$. The absolute gaps are indicated by the shaded area

frequency at the middle of the gap. In this way, the ratio becomes more meaningful compared to the gap width, $\Delta \omega$, itself. if the system is scaled up or down, the ratio remains invariant to size scaling.

The variation of the band gap is investigated based on the rotational asymmetry of the elliptic cylinders. The band gap structure can be controlled by rotating the elliptic $\mathrm{BaTiO}_{3}$ rods which modifies the geometric factor of a sonic crystal. Therefore, rotation of the rods will cause a geometric anisotropy on the sonic wave propagation through the structure. Figure 3 shows the results of the calculation for the normalized width of the lowest band gap as a function of the rotation angle $\theta$. The filling fraction for the calculation is fixed at approximately 0.47 . It
Fig. 1 a Transverse crosssection of the $2 \mathrm{D}$ square lattice consisting of elliptical $\mathrm{BaTiO}_{3}$ rods embedded in a polar liquid, b rotated rods by an angle $\theta$, c and the related first Brillouin zone (BZ) of the $2 \mathrm{D}$ square lattice
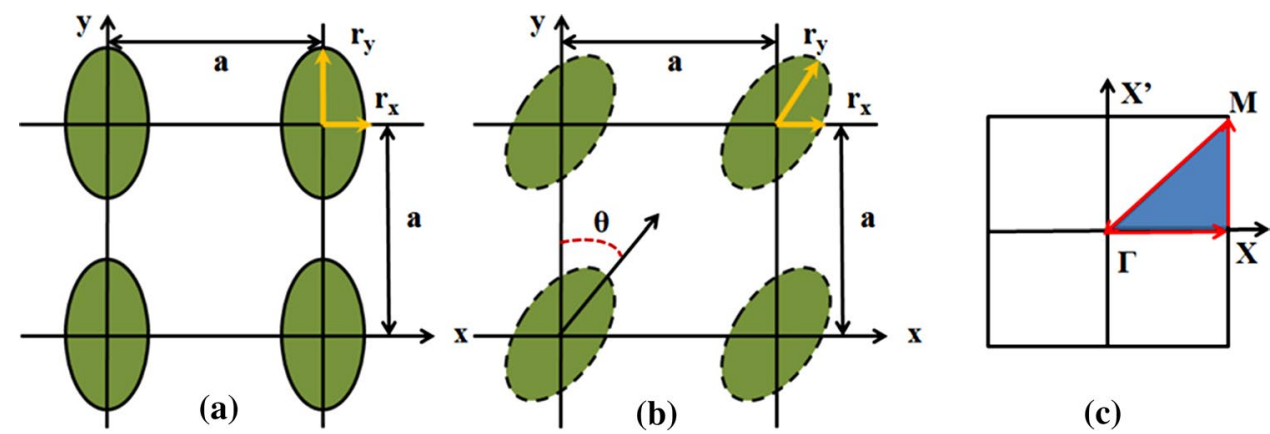

(c) 


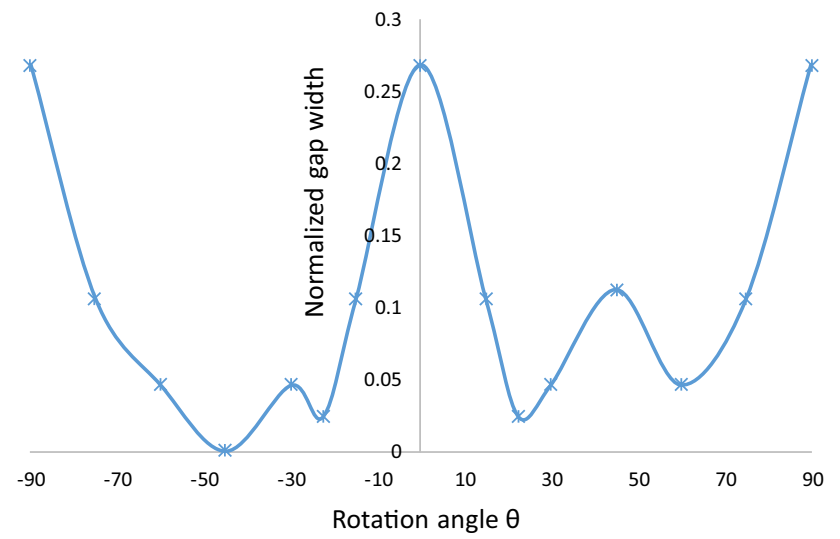

Fig. 3 The normalized gap-width $\Delta \omega / \omega_{\mathrm{g}}$ of the lowest band gap as the function of the rotation angle $\theta$

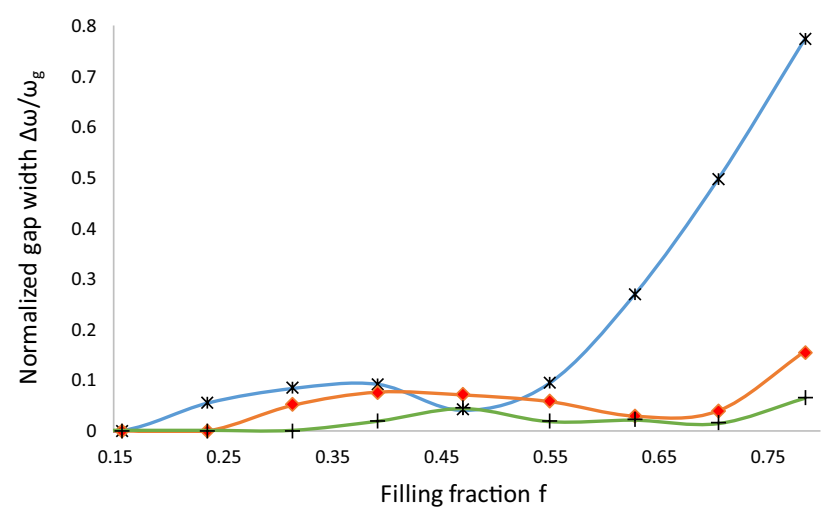

Fig. 4 The normalized gap-width $\Delta \omega / \omega_{\mathrm{g}}$ of the lowest three band gaps as a function of different filling fractions

must be noted that the largest value of the first band gap was found to be almost equal at $\theta=0^{\circ}$ and $\theta=90^{\circ}$.

To see the variation of the normalized gap-width $\Delta \omega / \omega_{\mathrm{g}}$ as a function of the filling factor $f$, the long axis of the elliptic rod is kept constant, and then the eccentricity is varied by changing the short axis. Figure 4 shows the $\Delta \omega / \omega_{\mathrm{g}}$ variation of the lowest three bands as a function of different filling fractions. The length of the major axis $r_{y}$ is fixed at $0.5 a_{\mathrm{o}}$ while the minor axis increment is set to $0.5 a_{\mathrm{o}}$ in $\Delta r_{x}$.

The highest symmetry was obtained from setting $r_{x}$ and $r_{y}$ to $r=0.5 a_{\mathrm{o}}$ yielding the largest normalized band width. Obviously, the shape of the rods becomes circular and the circle has the highest symmetry of all.

\section{Equi-frequency surface}

Reflection and refraction of a certain wave can be analyzed in the space of wave numbers by considering the equi-frequency surface of the wave (EFS). This surface is described by the dispersion relation of the anisotropic medium at the fixed frequency $\omega$. Therefore, the velocity of the propagating wave in an anisotropic medium can be obtained by the group velocity $\vec{v}_{\mathrm{g}}=\nabla_{\mathrm{k}} \omega(\vec{k})$. It is easy to imagine that a propagating wave in an isotropic medium with a given frequency $\omega o$ forms a spherical equi-frequency surface, which implies that the wave vector and group velocity vector are always in the same direction. However, equi-frequency surface is not always spherical. If the surface normal is not parallel to the wave vector, then the wave vector and the group velocity vector vg will be in different directions. The equi-frequency dependence inherits a physical meaning for the analysis of 2D geometries. The equi-frequency dependence simply describes all the possible waves with the given frequency $\omega$ and various wave vectors, the directions of the reflected and the refracted rays can be determined by simply finding the points in equi-frequency dependences of media that satisfy the momentum conservation law at a known orientation of the boundary and a given angle of incidence of the wave. Here, we present some numerical examples, which are significant for our PC structures.

The EFS shown in Fig. 5a of the suggested phononic crystal structure is obtained by calculating all the possible eigen frequencies over the irreducible BZ of the entire BZ. The possible solutions that are all allowed wave vectors in the first BZ forms a point on the surface. The plot is very informative, however, it is not easy to analyze. Figure $5 \mathrm{~b}$ shows contours of dispersion surfaces with values on the contours given. The equi-frequency contours (EFC) are not only easier to visualize all the possible wave vectors but also supplementary in analyzing behaviors of phononic crystals. The contours in the plot fundamentally represent the contours of the energy flow which may allow identification of refractive index irregularities. The circular contours in the central region of the plot in Fig. 5b imply that energy propagation has a directional symmetry. However, the EFC inevitably deviates from being circular and loses its shape as the contour evolves as a function of frequency. The circular contours are distorted and become a rectangular-like contour by leading to a simple symmetry for values of $k x$ equal to $k y$.

\section{Conclusions}

In this article, the acoustic wave propagation of a square lattice sonic crystal is studied. A sonic crystal made of elliptic cross-section of $\mathrm{BaTiO}_{3}$ rods in a polar liquid (water) was considered to investigate the existence of complete band gaps. The largest normalized gap-width $\Delta \omega /$ $\omega_{\mathrm{g}}$ of the lowest band gap as the function of the rotation angle was found to be the same at two different angles, $\theta=0^{\circ}$ and $\theta=90^{\circ}$. The existence of tunable band gaps in 


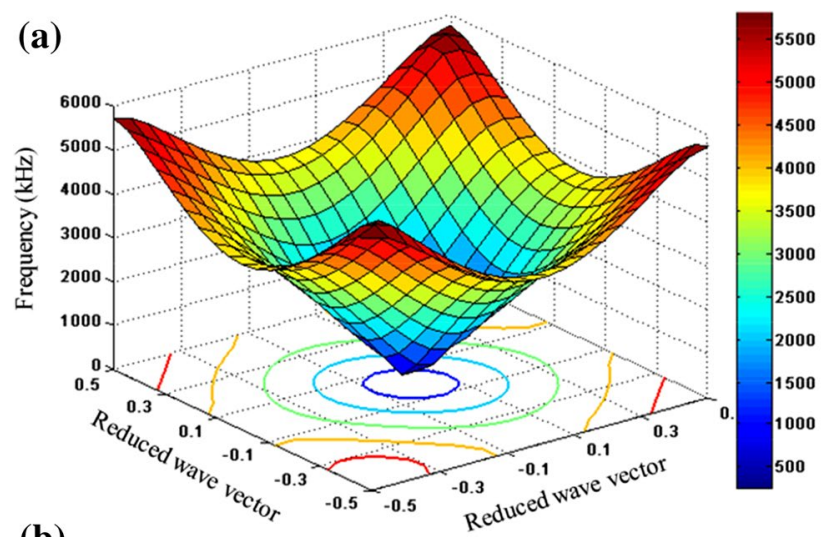

(b)

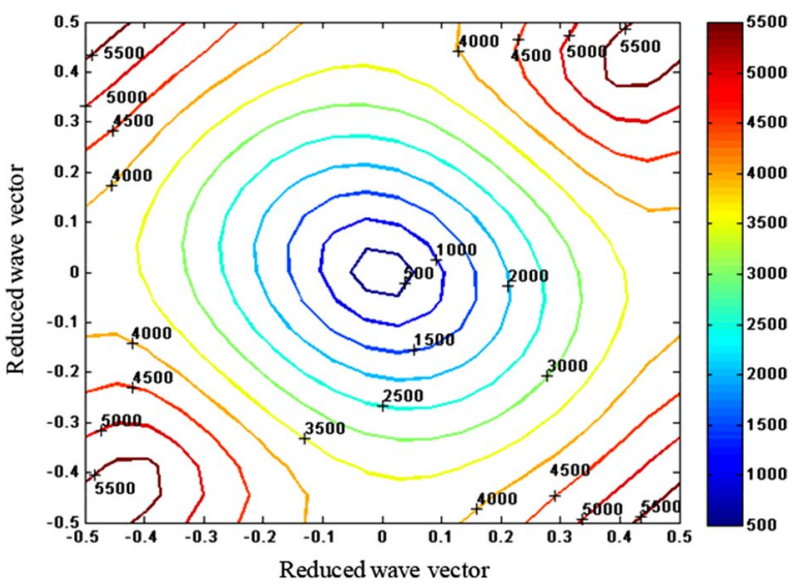

Fig. 5 a The band surface, $\omega(k)$, and $\mathbf{b}$ the equi-frequency contours for the lowest mode on the dispersion plot

the frequency regime are found. The appearance and disappearance of the band gaps imply rotational anisotropy for angles of $\pm 45^{\circ}$. The influence of filling fraction on the sonic eigenfrequencies was also taken into consideration. The study showed that when the fill factor reaches its maximum, the band gap widths were enlarged by opening up in the direction of lower and higher frequencies. Sonic crystal thus offers an alternative way towards the control and arbitrary manipulation of the sound waves. The findings may be useful for some of the applications of sonic crystal in acoustic devices, such as frequency filter, acoustic waveguides, metamaterials.

Acknowledgements The author (Ekmel Ozbay) acknowledges partial support from the Turkish Academy of Sciences.

\section{References}

1. M. Sigalas, M.S. Kushwaha, E.N. Economou, M. Kafesaki, I.E. Psarobas, W. Steurer, Classical vibrational modes in photonic lattices: theory and experiment. Z. Kristallogr 220, 765-809 (2005)
2. Y. Pennec, J. Vasseur, B. Djafari-Rouhani, L. Drobrzyski, P. Demier, Two-dimensional phononic crystals: examples and applications. Surf. Sci. Rep. 65(8), 229-291 (2010)

3. T. Miyashita, Sonic crystals and sonic wave-guides. Meas. Sci. Technol 16, 47-63 (2005)

4. X.D. Zhanga, Z.Y. Liu, Negative refraction of acoustic waves in two-dimensional phononic crystals. Appl. Phys. Lett. 85, 341-343 (2004)

5. M.S. Kushwaha, B. Djafari-Rouhani, Sonic-stop bands for periyodic arrays of metallics rods: honeycomb structure. J. Sound Vib 218(4), 697-709 (1998)

6. M. S. Kushwaha, P. Halevi, Stop bands for periodic metallic rods: sculpture that can filter the noise, Appl. Phys. Lett. 7, 3218-3220 (1997)

7. J.V. Sánchez-Pérez, C. Rubio, R. Martínez-Sala, R. SánchezGrandía, V. Gómez, Appl. Phys. Lett. 81(27), 5240 $5242(2002)$

8. R. Martínez-Sala, J. Sancho, J.V. Sánchez-Pérez, J. Llinares, F. Meseguer, Sound attenuation by sculpture. Nature 378, 241241 (1995)

9. J.V. Sánchez-Pérez, C. Rubio, R. Martínez-Sala, R. SánchezGrandía, V. Gómez, Acoustic barriers based on periodic arrays of scatterers. Appl. Phys. Lett. 81, 5240-5242 (2002)

10. C. Uris, H. Rubio, Estellés, J.V. Sánchez-Pérez, R. MartínezSala, J. Llinares, Design of lightweight multilayer partitions based on sonic crystals. Appl. Phys. Lett. 79, 4453-4454 (2001)

11. M. Kafesaki, M.M. Sigalas, N. Garcia, Frequency modulation in the transmitivity of wave guides in elastic band gap materials. Phys. Rev. Lett. 85, 4044-4047 (2000)

12. Z. Liu, X. Zhang, Y. Mao, Y.Y. Zhu, Z. Yang, C.T. Chan, P. Sheng, Locally resonant sonic materials. Science 289(5485), 1734-1736 (2000)

13. M.S. Kushwaha, P. Halevi, G. Martinez, L. Dobrzynski, B. Djafari-Rouhani, Theory of acoustic band structure of periodic elastic composites. Phys. Rev. B 49, 2313-2322 (1994)

14. J.O. Vasseur, B. Djafari-Rouhani, L. Dobrzynski, P.A. Deymier, Acoustic band gaps in fibre composite materials of boron-nitride structure. J. Phys.: Condens. Matter. 9, 7327

15. M.M. Sigalas, Theoretical study of three dimensional elastic band gaps with the finite difference time-domain method. J. Appl. Phys 87, 3122-3125 (2000)

16. V. Romero-García, J. V. Sánchez-Pérez, L. M. Garcia-Raffi, Evanescent modes in sonic crystals: complex dispersion relation and supercell approximation, J. Appl. Phys. 10, 044907 (2010)

17. V. Laude, Y. Achaoui, S. Benchabane, A. Khelif, Evanescent Bloch waves and the complex band structure of phononic crystals. Phys. Rev. B 80, 092301 (2009)

18. M. Kafesaki, E.N. Economou, Multiple scattering theory for 3D periodic acoustic composites. Phys. Rev. B 60, 11993-12001 (1999)

19. F.G. Wu, Z.L. Hou, Z.Y. Liu, Y.Y. Li, Point defect states in two-dimensional phononic crystals. Phys. Lett. A 292, 198-202 (2001)

20. J.V. Sánchez-Perez, D. Caballero, R. Martínez-Sala, C. Rubio, J. Sánchez Dehesa, F. Meseguer, J. LLinares, F. Gálvez, Sound attenuation by a two-dimensional array of rigid cylinders. Phys. Rev. Lett. 80, 5325-5328 (1998)

21. H. Pichard, O. Richoux, J.-P. Groby, Experimental demonstrations in audible frequency range of band gap tunability and negative refraction in two-dimensional sonic crystal. J. Acoust. Soc. Am 132(4), 2816-2822 (2012)

22. R. Wang, X. Wang, B. Gu, G. Yang, Effects of shapes and orientations of scatterers and lattice symmetries on the photonic band gap in two dimensional photonic crystals. J. Appl. Phys 90, 4307-4013 (2001) 
23. S.A. El-Naggar, S.I. Mostafa, N.H. Rafat, Complete band gaps of phononic crystal plates with square rods. Ultrasonics 52, 536542 (2012)

24. H. Salehi, M. Aryadoust, M. Z. Shoushtari, The generalization of structure factor for rods by polygon section in two-dimensional phononic crystals, IJST 37A4, 457-462 (2013)

25. X. Li, F. Wu, H. Hu, S. Zhong, Y. Liu, Large acoustic band gaps created by rotating square rods in two-dimensional periodic composites. J. Phys. D: Appl. Phys 36, L15-L17 (2003)

26. R.F. Probstein, Physicochemical hydrodynamics: an introduction (Butterworth Publishers, MA, 1989)

27. A.R. Johnston, Dispersion of electro-optic effect in $\mathrm{BaTiO}_{3}$. J. Appl. Phys 42(9), 3501-3507 (1971)

28. Y.-Z. Wang, F.-M. Lia, W.-H. Huanga, X. Jiangc, Y.-S. Wangd, K. Kishimotob, Wave band gaps in two-dimensional piezoelectric/piezomagnetic phononic crystals. Int. J. Solids Struct $\mathbf{4 5}$, 4203-4210 (2008)

29. X.-K. He, L.-B. Zeng, Q.-S. Wu, L.-Y. Zhang, K. Zhu, Y.-L. Liu, Determination of elastic, piezoelectric, and dielectric constants of an $\mathrm{R}$ : $\mathrm{BaTiO}_{3}$ single crystal by Brillouin scattering. Chin. Phys. B 21(6), 067801-067805 (2012)

30. C. Renu, Structural, Elastic and Thermal Properties of $\mathrm{BaTiO}_{3}$. Invert. J. Sci. Technol. 7(2), 72-77 (2014)

31. S. Piskunov, E. Heifets, R.I. Eglitis, G. Borstel, Bulk properties and electronic structure of $\mathrm{SrTiO}_{3}, \mathrm{BaTiO}_{3}, \mathrm{PbTiO}_{3}$ perovskites: an ab initio HF/DFT study. Comp. Mater. Sci 29, 165-178 (2004)

32. T. Miyashita, Sonic crystals and sonic wave-guides. Meas. Sci. Technol 16, R47-R63 (2005)

33. L.-Y. Wu, L.-W. Chen, The dispersion characteristics of sonic crystals consisting of elliptic cylinders. J. Phys. D. Appl. Phys 40, 7579-7583 (2007)

34. D. P Elford, Band gap formation in acoustically resonant phononic crystals. $\mathrm{PhD}$ thesis. Loughborough University Institutional Repository, 2010

35. R.H. Lipson, C. Lu, Photonic cr ystals: a unique partnership between light and matter. Eur. J. Phys 30, S33-S48 (2009) 